\title{
Cystic Squamous Metaplasia in Benign Phyllodes Tumor
}

\author{
Md Ali Osama, Arupparna Sen Gupta and Seema Rao*
}

Department of Histopathology, Sir Gangaram Hospital, New Delhi

\section{Case History}

A 37-year-old female patient presented to the Department of Surgical Oncology with a history of palpable mass in right breast of 6 months' duration. Physical examination revealed a firm, palpable, mobile mass of approximately $6 \times 5 \mathrm{~cm}$ size at 2-4 o'clock position. The mass was not fixed to the underlying muscle or the overlying skin. There was no history of trauma or any constitutional symptom. Ultrasound [Fig 1a] and mammographic [Fig 1b] evaluation was highly suspicious of malignancy (BIRADS 4C), and hence excisional biopsy was recommended. Grossly, the lesion was received as two fibrofatty tissue pieces measuring $6 \times 5 \times 4 \mathrm{~cm}$ and $5 \times 3 \times 2 \mathrm{~cm}$. Cut surface showed a well circumscribed tumor which was grey white, firm with regular borders. Within the tumor mass, multiple well-defined cysts varying in size from 0.2 to $1.5 \mathrm{~cm}$ were identified which were filled with thick whitish material [Fig 2]. Microscopic examination showed a well circumscribed biphasic tumor composed of epithelial and stromal elements. The epithelial elements were lined by bland looking epithelial-myoepithelial bilayer. The stroma showed moderate cellularity and was composed of spindle shaped fibroblastic cells arranged in fascicles. Focal adenosis along with occasional foci of usual ductal hyperplasia were observed in the epithelial component [Fig 3a]. No stromal overgrowth, cellular atypia or increased mitotic activity was observed. In addition, many variably sized cystic spaces lined by squamous epithelium were noted. These cystic spaces were filled with laminated keratin material [Fig 3b, c and d]. By these histopathological findings, the case was diagnosed as Benign phyllodes tumor with cystic squamous metaplasia. The patients showed no signs of recurrence or metastasis on follow up of 2 years' duration.

\section{Discussion}

Phyllodes tumors are rare fibroepithelial neoplasms and represent 0.5 to $1.5 \%$ of breast tumors ${ }^{[1]}$. They usually occur in females aged 45 to 49 years. World Health Organization (WHO) classified Phyllodes tumors into benign, borderline and malignant categories on the basis of a constellation of histological parameters, i.e., the degree of stromal cellularity and atypia, mitotic count per
10 high power fields, stromal overgrowth, tumor borders and presence of heterologous stromal element. Grading of phyllodes tumors is clinically relevant as it helps to predict the clinical behavior: benign tumors have the ability to recur locally; borderline tumors in addition to local recurrence also have a low risk of metastasis while the malignant ones have the highest risk of metastatic behavior. As a result, these tumors are subjected to complete local excision. Metaplastic changes may be observed both in the epithelial or stromal components of the tumor. The stroma may show chondroid, osteoid, or lipomatous metaplasia while the epithelium occasionally shows apocrine or squamous metaplasia ${ }^{[2]}$. Although, varying degrees of squamous metaplasia has been reported in about $10 \%$ of phyllodes tumor ${ }^{[2]}$; however cystic squamous metaplasia is a relative rare finding. Tan et al, in their case series of 335 phyllodes tumors found epithelial squamous metaplasia in 12 cases, $5(1.5 \%)$ of which revealed squamous cysts ${ }^{[3]}$. Apart from phyllodes tumor, squamous metaplasia can also occur in a variety of other benign and malignant breast lesions. Some of the benign lesions include fibroepithelial tumors, intraductal papilloma, duct ectasia, subareolar abscess and inflamed breast cysts. The malignant ones include metaplastic carcinoma, adenosquamous carcinoma and squamous cell carcinoma. Examination of cystic areas of squamous metaplasia by aspiration cytology may then lead to misdiagnosis, squamous cyst being most common error ${ }^{[4]}$.

\section{Conclusion}

Extensive cystic squamous metaplasia is a rare feature of phyllodes tumor. Examination of cystic areas of squamous metaplasia by aspiration cytology may be confounding and lead to a misdiagnosis. This uncommon condition poses a diagnostic dilemma and hence clinicians, radiologists and pathologists must be aware of this entity to avoid misinterpretation. Hence, this case highlighted a unique histomorphology in phyllodes tumor.

\section{Funding \\ None}

\section{Competing Interests}

None 


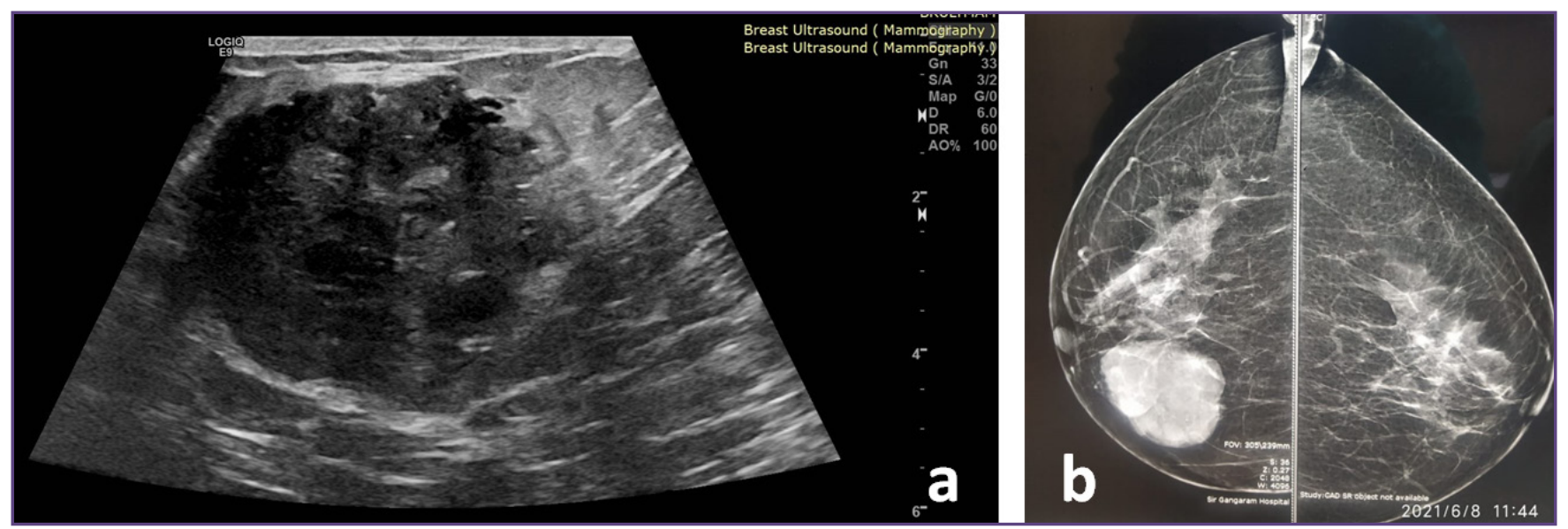

Fig. 1: a: Ultrasound image shows a lobulated mass with heterogeneous echotexture and cystic changes. b: Craniocaudal mammogram shows a large, well defined, lobulated, homogenously dense mass.

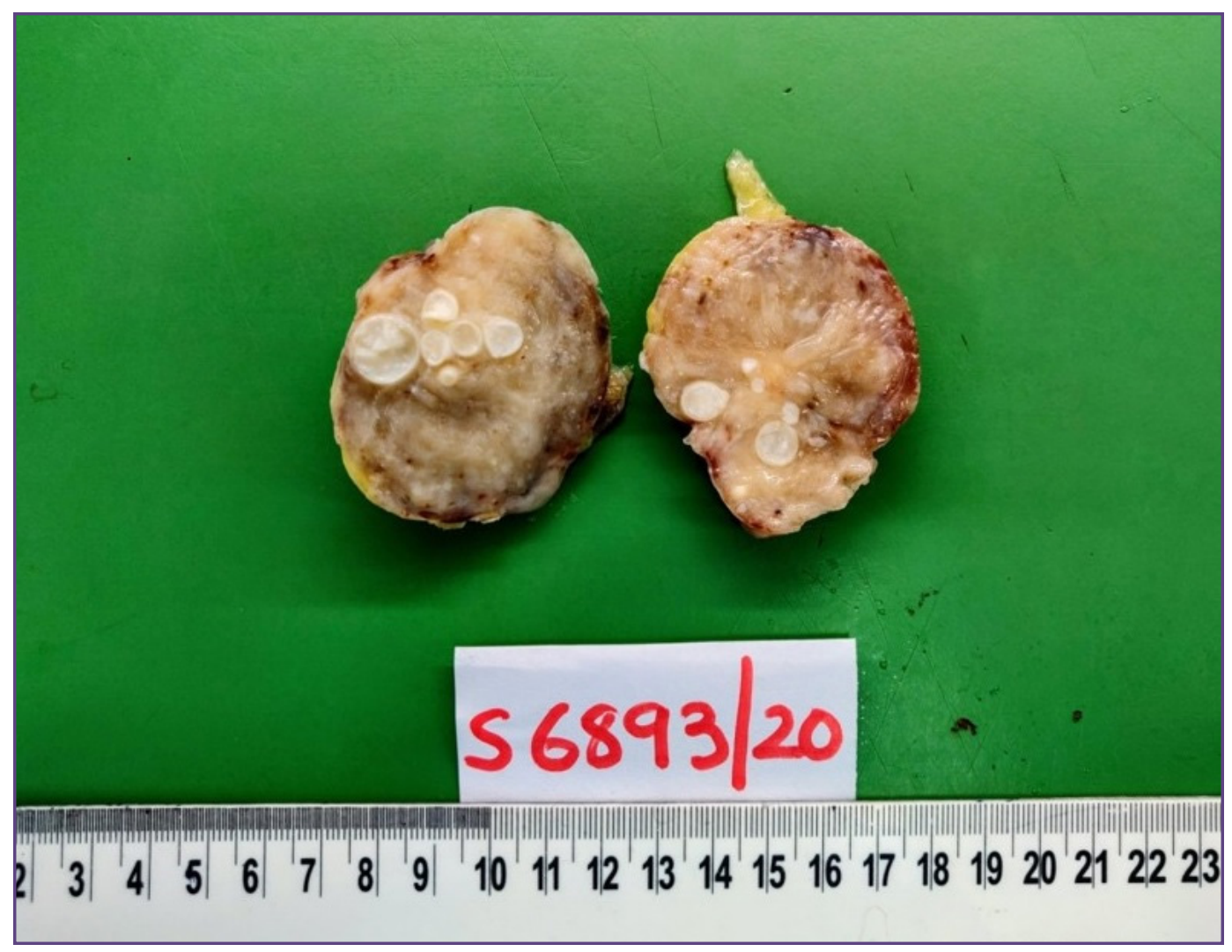

Fig. 2: Gross examination of tumor showing cystic spaces filled with thick whitish material. 

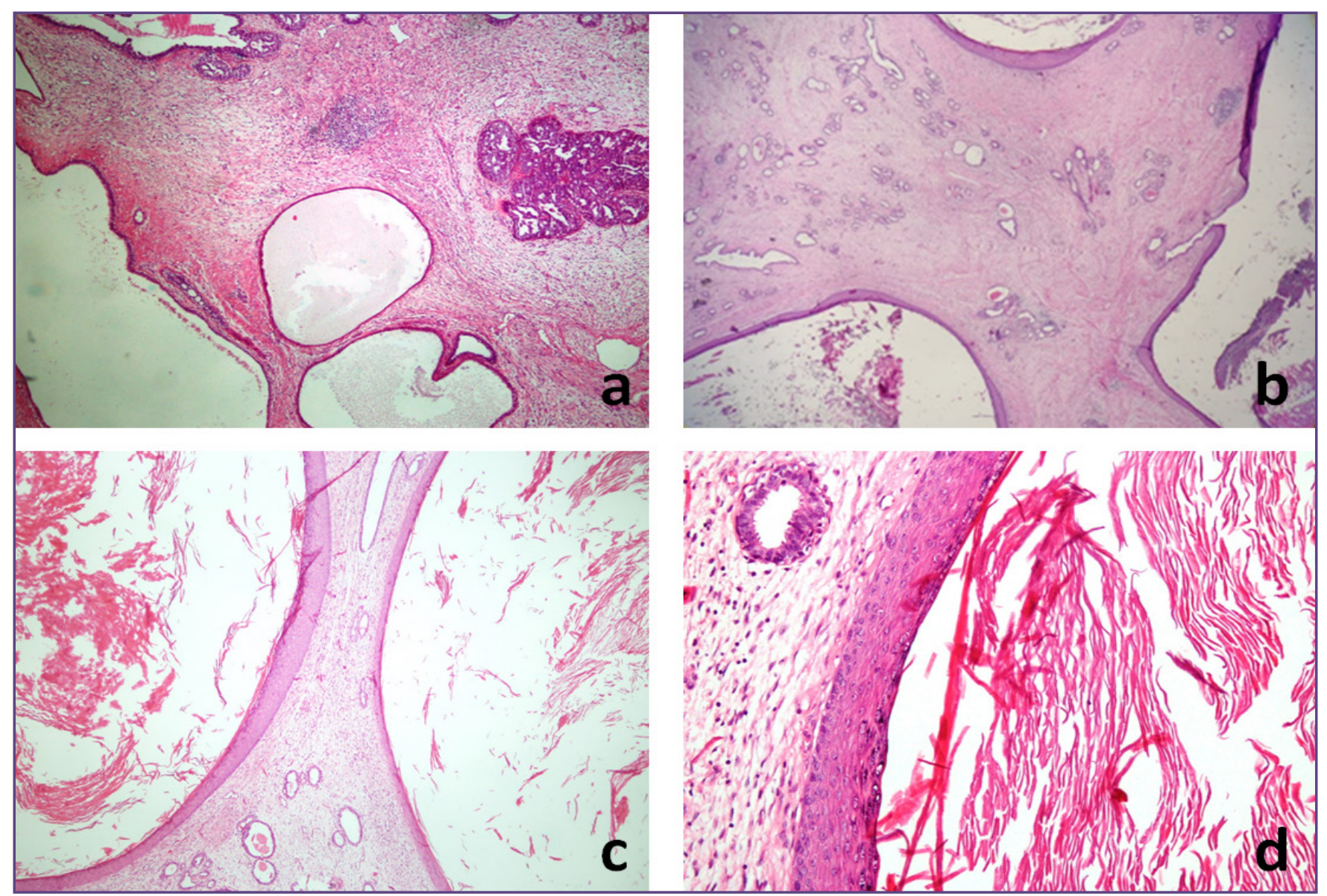

Fig. 3: a: Microscopic examination shows benign phyllodes tumor composed of epithelial and stromal elements with occasional focus of usual ductal hyperplasia (HE 200x), b: Microscopic examination shows multiple intratumoral cystic spaces lined by stratified squamous epithelium (HE 100x). c: Intratumoral cystic spaces are lined by stratified squamous epithelium and filled with thick laminated keratinous material (HE 200x). d: Cystic space lined by stratified squamous epithelium and filled with laminated keratinous material (HE 400x).

\section{Reference}

1. Chaney AW, Pollack A, McNeese MD, et al. Primary treatment of cystosarcoma phyllodes of the breast. Cancer. 2000;89:1502-11.

2. Rosen PP. Fibroepithelial neoplasms. In: Rosen's Breast Pathology. 2nd ed. Philadelphia, PA: Lippincott Williams \& Wilkins; 2001:163-200.
3. Tan PH, Jayabaskar T, Chuah KL, et al. Phyllodes tumors of the breast: the role of pathologic parameters. Am J Clin Pathol. 2005;123:529-40.

4. Chakrabarti I, Agarwala P, Bera P, Bhaduri S. Benign Phyllodes Tumor With Cystic Squamous Metaplasia: A Cytohistological Correlation of a Rare Case. Iran J Pathol. 2017;12:291-4.

\section{*Corresponding author:}

Dr Seema Rao, Department of Histopathology, Sir Gangaram Hospital, New Delhi, India

Phone: +91 9868551374

Email: seemarao1974@yahoo.co.in

Financial or other Competing Interests: None.

Date of Submission $\quad: 14 / 06 / 2021$

Date of Final Revision : 10/07/2021

Date of Acceptance $\quad: \quad 18 / 07 / 2021$

Date of Publication $\quad: 30 / 07 / 2021$ 